\title{
Ethnobotanical study of plants used in management of livestock health problems by Afar people of Ada'ar District, Afar Regional State, Ethiopia
}

\author{
Mirutse Giday ${ }^{*}$ and Tilahun Teklehaymanot
}

\begin{abstract}
Background: The great majority of the Afar people of Ethiopia are pastoralists, highly dependent on livestock and livestock products. Livestock productivity is, however, frequently affected by different diseases. Although many districts in the Region have veterinary clinics, they lack basic facilities. As a result, the Afar people are still dependent on local materials, mainly plants, and traditional knowledge to manage livestock health problems. However, there is a serious threat to such local resources mainly due to recurrent drought and influence of modernization. Hence there is a need for proper documentation and evaluation of the existing ethnoveterinary knowledge in the Region. This study was aimed at documenting and analysing ethnoveterinary knowledge of people in Ada'ar District of the Afar Region associated with the use of plants.
\end{abstract}

Methods: The study involved interviewing selected knowledgeable Afar people in Ada'ar District on the use of plants to manage livestock ailments. Fidelity Level (FL) values were calculated for the reported medicinal plant to estimate their healing potentials. Specimens of reported medicinal plant were collected, identified and deposited at the National Herbarium, Addis Ababa University.

Results: The study revealed 49 medicinal plants as being used by the Afar people of Ada'ar District for the treatment of various livestock ailments, the majority of which (67.3\%) were shrubs. Highest number of medicinal plants was used to treat blackleg, contagious caprine pleuropneumonia (CCPP), sudden sickness and pneumonia. Leaf was the most frequently sought plant part, accounting for $47 \%$ of the reported plants. All the medicnal plants used in the District were uncultivated ones growing in semi-disturbed and disturbed habitats as remnant plants and weeds. Cissus quadrangularis and Solanum incanum were the plants scoring the highest fidelity level values for their use to treat blackleg and respiratory tract problems, respectively.

Conclusion: The study revealed that there is still rich knowledge of ethnoveterinary medicine in Ada'ar District. There was no habit of cultivating medicinal plants by people in the study area. Efforts, should, therefore, be made to protect these medicinal plants from further depletion, especially those that are scarcely availabale. Better attention should be given to medicinal plants with the highest fidelity level values as such values could indicate potencies of the plants.

Keywords: Medicinal plants, Ethnoveterinary practices, Afar, Ada'ar District, Ethiopia

\footnotetext{
* Correspondence: mirutseg@yahoo.com

Aklilu Lemma Institute of Pathobiology, Addis Ababa University, P.O. Box

1176, Addis Ababa, Ethiopia
} 


\section{Background}

Ethiopia is among the countries in Africa with the highest livestock populations [1]. Livestock production is an integral part of the Ethiopian agriculture and shares about $40 \%$ of the total agricultural output [2]. Although the country is rich in its livestock population, it is among the countries in the world with the lowest unit output. The poor health condition of its livestock has partially been responsible for the low productivity.

Modern livestock health care is still at its lowest stage in the country due to lack of adequate clinics, veterinarians and supply of drugs. Besides, most modern drugs are expensive and, as a result, not affordable by the majority of Ethiopian farmers and pastoralists. As a result, people rely on their traditional knowledge, practices and locally available materials (mainly plants) in the management of diseases of their domestic animals. Ethnoveterinary practice in the country is, however, being affected due to acculturation and depletion of plants as a result of environmental degradation, deforestation and over exploitation of the medicinal plants themselves. However, very little of the ethnoveternary knowledge in Ethiopia in relation to the use of medicinal plants is so far properly documented and analysed [3-8].

The Afar people of Ethiopia reside in the Afar Regional State. The great majority of them are pastoralists and, as a result, highly dependent on livestock and livestock products. Livestock productivity is, however, frequently affected by different diseases. They, therefore, give high priority to the well being of their domestic animals. As access to modern veterinary facilities in the Afar Region is very limited, the people are still expected to largely rely on their traditional knowledge in the management of livestock health problems.

To the author's knowledge, there is only one proper ethnobotanical study conducted in the Afar region [9]. The current study tried to document and analyse ethnoveterinary knowledge and practices of the pastoral Afar people residing in Ada'ar District of the Afar Region with emphasis on medicinal plants. Ada'ar is among the districts in the Region with the highest populations of cattle, goats and sheep.

\section{Materials and methods}

\section{The Afar Region and people}

The Afar people reside in the Afar Regional State, northeastern Ethiopia. Afar is one of the major pastoral regions in Ethiopia. It shares borders with Eritrea in the north, Djibouti and Somali Region in the east, Tigray and Amhara regions in the west and Oromiya Region in the south. Semera is the administrative town of the Region and is located at about $600 \mathrm{~km}$ northeast of Addis Ababa. The Afar Region is divided into five zones, 29 districts (weredas) and 355 kebeles [10]. Kebele is the smallest administrative unit in Ethiopia. Altitude in the Region ranges from $1500 \mathrm{~m}$ a.s.l. to $120 \mathrm{~m}$ below sea level [10]. The Region receives bimodal rainfall with a precipitation of $150-500 \mathrm{~mm}$ per annum and temperature varies from 20 to $48^{\circ} \mathrm{C}[10]$.

Based on the 1994 national census, the population of the Afar people of Ethiopia was projected to be 1.3 million in 2004, of which 56\% are males and $44 \%$ females [11]. Afar economy is highly dependent on livestock and livestock products. The livestock population of the Afar Region is estimated to be about 4 million [10]. Cattle, sheep, goats, camels and donkeys are commonly raised. Livestock productivity is, however, affected by different diseases that occur in the Region [12]. Although the majority of districts in the region have veterinary clinics, they lack basic facilities such as electricity, water and clinical and diagnostic equipment and most of them frequently face shortage of drugs [10]. Afar people residing in districts adjacent to the neighbouring highlands of the Tigray and Amhara regions cultivate sorghum, maize, teff and cotton [10].

\section{The study district}

The current study was conducted in Ada'ar District belonging to Zone 1 of the Afar Region of Ethiopia (Figure 1). The District is bordered by Mile (east), Chifra (northwest) and Dewe (southeast) districts of the Afar Region and Bati (west) and Telalak (southwest) districts of the Amhara Region. The District is administratively divided into 13 kebeles. According to Ada'ar District Administration, the total human population of the District is 59,637. According to Ada'ar District Pastoral, Agriculture and Rural Development Office, livestock population of the District in 2009 was estimated to be 683,977 , of which cattle had the highest population $(166,964)$, followed by sheep $(164,857)$ and goat $(329,714)$. The same Office reported that anthrax, blackleg and pastereulosis were among the most prevalent livestock ailments in the District. There are three veterinary health posts and two private drug shops in the District.

\section{Selection of informants}

For the interview, a total of 21 knowledgeable Afar informants (20 males and 1 female) from eight kebeles, namely Burka, Neile-Askala, Siylu-Waky, Ada'ar, Ledi, Woantu-Fursa, Eluwuha and Jeldi were involved. All the informants were Muslims between the ages of 24 and 68 years. The informants were identified with help of Ada'ar Administration Office and elders nominated by the same office.

\section{Data collection method and type of data collected}

Ethnobotanical data were gathered mainly through individual interviews conducted with the knowledgeable informants during five trips made to the study area 


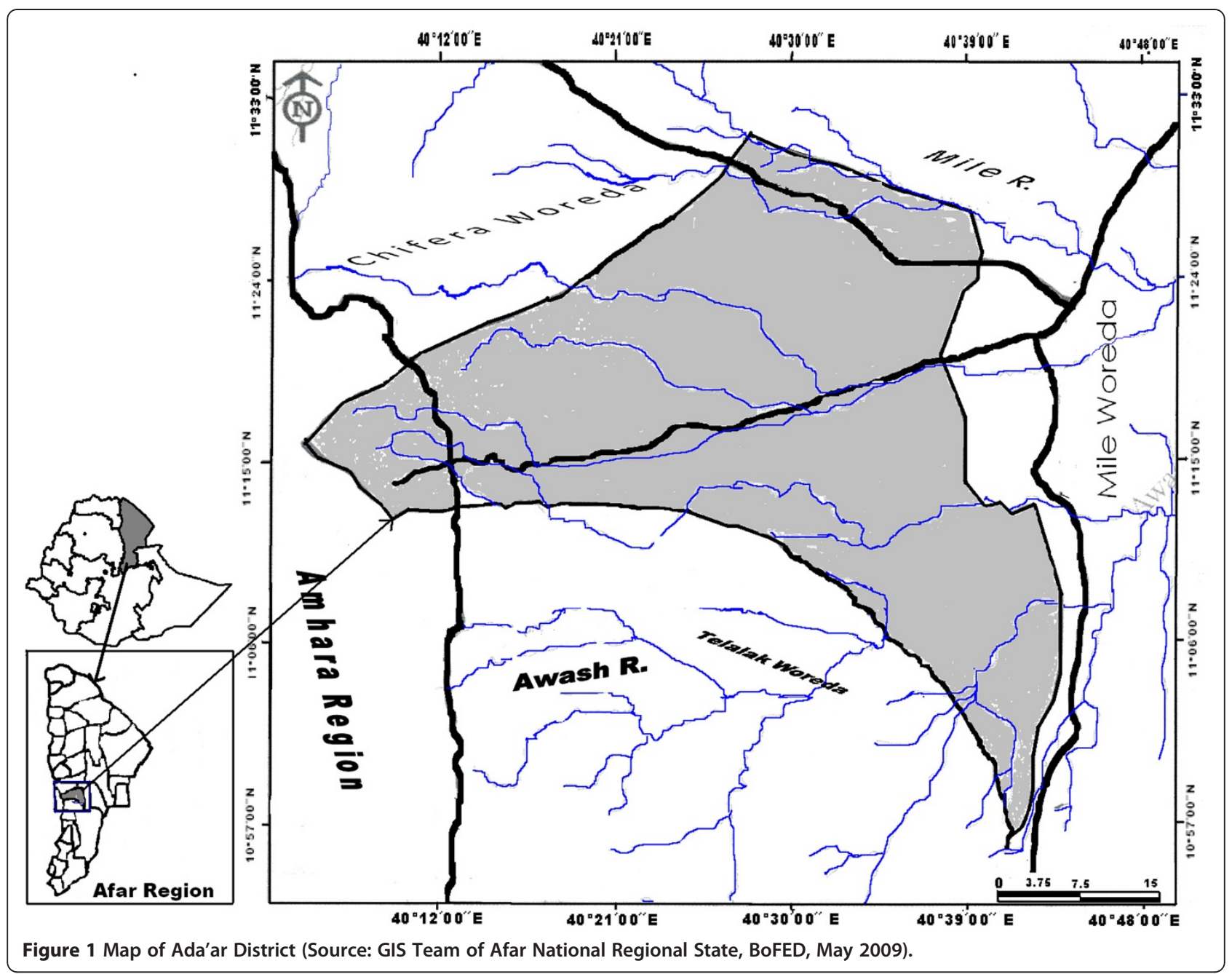

between August 2008 and April 2011. For this purpose, a semi-structured interview guide was prepared in English beforehand. Interviews with informants were conducted by the researchers with the help of Afar translators. During interview, data regarding the kinds of livestock ailments treated or prevented and the types of medicinal plants used (including their local names), the plant parts used, modes of remedy preparations, route of administration and dosages were collected. Besides, information in relation to local trade, cultivation practices, habitat, abundance and threats of claimed medicinal plants was gathered. Specimens of the reported medicinal plant were collected, dried and identified by their scientific name, and vouchers were deposited at the National Herbarium, Addis Ababa University.

\section{Data analyzing methods}

Microsoft Excel spreadsheet software was employed for organising and analysis ethnobotanical data. Relative importance (RI) of each cited medicinal plant was calculated using a method by Bennett and Prance [13]. RI is calculated using the formula RI = NP + NCS, where NP is obtained by dividing the number of properties (reported specific ailments) attributed to a species divided by the total number of properties attributed to the most versatile species (species with the highest number of properties). NCS is the number of body systems (ailment categories) treated by a given species divided by the total number of body systems treated by the most versatile species. Species with RI value of 2.0 (the highest possible value) are the ones with the highest diversity of medicinal application.

Relative healing potential of each medicinal plant reported was estimated using an index called fidelity level (FL) based on the proportion of informants who agreed on the use of a given medicinal plant against a given major ailment category. The formula for $\mathrm{FL}$ is given as $F L=I_{p} / I_{u} \times 100$, where $I_{p}$ is the number of informants who independently indicated the use of a species for the same major ailment and $I_{u}$ the total 
Table 1 Medicinal plants of the Afar people in Ada'ar District

\begin{tabular}{|c|c|c|c|c|c|c|c|c|c|}
\hline Scientific name & Family & $\begin{array}{l}\text { Local } \\
\text { name }\end{array}$ & Habit & Part used & $\begin{array}{l}\text { Local } \\
\text { disease } \\
\text { name }\end{array}$ & $\begin{array}{l}\text { English disease } \\
\text { name }\end{array}$ & $\begin{array}{l}\text { Animal } \\
\text { treated }\end{array}$ & $\begin{array}{l}\text { Administration } \\
\text { route }\end{array}$ & $\begin{array}{l}\text { Voucher } \\
\text { No. }\end{array}$ \\
\hline \multirow{2}{*}{$\begin{array}{l}\text { Acacia nilotica (L.) Willd. ex } \\
\text { Del. }\end{array}$} & Fabaceae & kenselto & tree & fruit & gublo & $C C P^{1} P$ & goat & oral & MG-TT-11 \\
\hline & & & & & uruga & diarrhea & $\begin{array}{l}\text { Goat, } \\
\text { sheep }\end{array}$ & oral, nasal & \\
\hline $\begin{array}{l}\text { Acacia oerfota (Forssk.) } \\
\text { Schweinf. }\end{array}$ & Fabaceae & goronto & shrub & bark & geno & $\begin{array}{l}\text { sudden } \\
\text { sickness }\end{array}$ & cattle & oral, nasal & $\begin{array}{l}\text { MG-TT-14, } \\
\text { MG-TT-5 }\end{array}$ \\
\hline Acalypha fruticosa Forssk. & Euphorbiaceae & subahi & shrub & leaf & indahi & CCPP & sheep & oral & MG-TT-12 \\
\hline \multirow[t]{2}{*}{ Acalypha indica $\mathrm{L}$. } & Euphorbiaceae & $\begin{array}{l}\text { baro } \\
\text { berbere }\end{array}$ & herb & $\begin{array}{l}\text { whole } \\
\text { part }\end{array}$ & geno & anthrax & $\begin{array}{l}\text { cattle, } \\
\text { camel }\end{array}$ & oral & MG-TT-38 \\
\hline & & & & leaf & ladore & blackleg & cattle & oral & \\
\hline $\begin{array}{l}\text { Aerva javanica (Burm.f) } \\
\text { Schultes }\end{array}$ & Amaranthaceae & alyaito & shrub & root & intibiaki & $\begin{array}{l}\text { ophthalmic } \\
\text { infection }\end{array}$ & goat & ophthalmic & MG-TT-10 \\
\hline \multirow[t]{3}{*}{ Aloe trichosantha Berger } & Aloaceae & ureita & shrub & leaf & geno & anthrax & goat & oral, nasal & MG-TT-15 \\
\hline & & & & leaf & gublo & CCPP & goat & oral, nasal & \\
\hline & & & & leaf & gublo & $\mathrm{CBPP}^{2}$ & cattle & oral, nasal & \\
\hline Argemone mexicana $\mathrm{L}$. & Solanaceae & baro bangi & herb & leaf & abeb & $\mathrm{FMD}^{3}$ & cattle & oral & MG-TT-45 \\
\hline \multirow{7}{*}{$\begin{array}{l}\text { Balanites aegyptiaca (L.) } \\
\text { Del. }\end{array}$} & Balanitaceae & uda & tree & root & finoita & bloat & animals & oral & MG-TT-54 \\
\hline & & & & root & geno & anthrax & $\begin{array}{l}\text { cattle, } \\
\text { goat, } \\
\text { camel }\end{array}$ & oral & \\
\hline & & & & root & geno & colic & cattle & oral, nasal & \\
\hline & & & & $\begin{array}{l}\text { above } \\
\text { ground }\end{array}$ & geno & $\begin{array}{l}\text { sudden } \\
\text { sickness }\end{array}$ & $\begin{array}{l}\text { goat, } \\
\text { cattle }\end{array}$ & oral, nasal & \\
\hline & & & & root & geremole & trypanosomiasis & camel & oral, nasal & \\
\hline & & & & root & indahi & CCPP & goat & oral, nasal & MG-TT-19 \\
\hline & & & & root & sole & diarrhoea & cattle & oral, nasal & MG-TT-2 \\
\hline $\begin{array}{l}\text { Balanites rotundifolia } \\
\text { (van Tieghem) Blatter }\end{array}$ & Balanitaceae & uda & shrub & root bark & kahiw & pneumonia & camel & nasal & MG-TT-49 \\
\hline Barleria acanthoides Vahl & Acanthaceae & ganselto & shrub & root & harayiti & blackleg & cattle & $\begin{array}{l}\text { oral, nasal, } \\
\text { auricular, } \\
\text { ophthalmic }\end{array}$ & MG-TT-47 \\
\hline \multirow[t]{3}{*}{ Boscia coriacea Pax } & Capparidaceae & urma & shrub & leaf & abeli & babesia & cattle & nasal & MG-TT-24 \\
\hline & & & & leaf & geno & anthrax & cattle & nasal & \\
\hline & & & & leaf & ladore & blackleg & cattle & oral, nasal & $\begin{array}{l}\text { MG-TT-1, } \\
\text { MG-TT-22 }\end{array}$ \\
\hline $\begin{array}{l}\text { Boscia senegalensis Lam. } \\
\text { ex Poiret }\end{array}$ & Capparidaceae & aitineba & shrub & leaf \& fruit & arba & tympanic bloat & cattle & oral & MG-TT-48 \\
\hline \multirow{2}{*}{$\begin{array}{l}\text { Bourreria orbicularis } \\
\text { (Hutch. \& E.A. Bruce) } \\
\text { Thulin }\end{array}$} & Boraginaceae & ulageita & shrub & leaf, bark & dale & wound & animals & $\begin{array}{l}\text { local on wound, } \\
\text { oral }\end{array}$ & MG-TT-16 \\
\hline & & & & leaf \& bark & harayita & blackleg & cattle & oral, nasal & \\
\hline Cadaba farinosa Forssk. & Capparidaceae & dinibayto & shrub & root & lahi biyak & pastereulosis & cattle & $\begin{array}{l}\text { oral, nasal, } \\
\text { auricular }\end{array}$ & MG-TT-29 \\
\hline \multirow[t]{2}{*}{ Cadaba glandulosa Forssk. } & Capparidaceae & udodoita & shrub & leaf & geno & pneumonia & cattle & oral, nasal & MG-TT-30 \\
\hline & & & & leaf & uruga & diarrhoea & cattle & oral, nasal & \\
\hline \multirow[t]{2}{*}{ Cadaba rotundifolia Forssk. } & Capparidaceae & angelita & shrub & leaf & ladore & blackleg & cattle & oral, nasal & $\begin{array}{l}\text { MG-TT-07, } \\
\text { MG-TT-25 }\end{array}$ \\
\hline & Apocynaceae & gela'eto & shrub & & abel & babesia & & & MG-TT-33 \\
\hline
\end{tabular}


Table 1 Medicinal plants of the Afar people in Ada'ar District (Continued)

\begin{tabular}{|c|c|c|c|c|c|c|c|c|c|}
\hline \multirow[t]{4}{*}{$\begin{array}{l}\text { Calotropis procera (Ait.) } \\
\text { Aitf. }\end{array}$} & & & & $\begin{array}{l}\text { branch, } \\
\text { seed }\end{array}$ & & & $\begin{array}{l}\text { goat, } \\
\text { sheep }\end{array}$ & $\begin{array}{l}\text { nasal, auricular, } \\
\text { oral }\end{array}$ & \\
\hline & & & & latex & aray mude & blackleg & cattle & local on legs & \\
\hline & & & & root & geno & colic & cattle & oral, nasal & \\
\hline & & & & latex & - & $\begin{array}{l}\text { prophylaxis for } \\
\text { different } \\
\text { diseases }\end{array}$ & cattle & local on skin & \\
\hline \multirow[t]{3}{*}{ Caralluma sp } & Asclepiadaceae & uramo & herb & $\begin{array}{l}\text { leaf, stem, } \\
\text { whole } \\
\text { part, } \\
\text { branch }\end{array}$ & $\begin{array}{l}\text { ladore, } \\
\text { haraimude }\end{array}$ & blackleg & cattle & $\begin{array}{l}\text { oral, nasal, } \\
\text { auricular }\end{array}$ & MG-TT-17 \\
\hline & & & & $\begin{array}{l}\text { above } \\
\text { ground }\end{array}$ & lahi biyak & pastereulosis & cattle & $\begin{array}{l}\text { oral, nasal, } \\
\text { auricular }\end{array}$ & \\
\hline & & & & flower & gublo & CBPP & cattle & oral, nasal & \\
\hline Cissus quadrangularis $\mathrm{L}$. & Vitaceae & $\begin{array}{l}\text { Musriga, } \\
\text { yaey'eto }\end{array}$ & climber & $\begin{array}{l}\text { leaf, } \\
\text { above } \\
\text { ground, } \\
\text { stem }\end{array}$ & $\begin{array}{l}\text { Harayiti, } \\
\text { ladore }\end{array}$ & blackleg & cattle & $\begin{array}{l}\text { oral, nasal, } \\
\text { auricular }\end{array}$ & $\begin{array}{l}\text { MG-TT-9, } \\
\text { MG-TT-26 }\end{array}$ \\
\hline \multirow[t]{2}{*}{$\begin{array}{l}\text { Citrullus colocynthis (L.) } \\
\text { Schrad. }\end{array}$} & Cucurbitaceae & dearteba & $\begin{array}{l}\text { trailing } \\
\text { herb }\end{array}$ & fruit & kehu & pneumonia & camel & $\begin{array}{l}\text { oral, nasal, } \\
\text { auricular }\end{array}$ & \\
\hline & & & & root \& leaf & sengite & CBPP & $\begin{array}{l}\text { cattle, } \\
\text { goat }\end{array}$ & oral \& nasal & MG-TT-8 \\
\hline $\begin{array}{l}\text { Cocculus pendulus } \\
\text { (J.R. \& G. Forst) Diels }\end{array}$ & Menspermaceae & hayofto & climber & root & finoita & bloat & animals & oral & MG-TT-50 \\
\hline $\begin{array}{l}\text { Commicarpus helenae } \\
\text { (J.A. Schultes) Meikle }\end{array}$ & Nyctaginaceae & kerbeti & herb & $\begin{array}{l}\text { whole } \\
\text { part }\end{array}$ & abeli & babesia & goat & $\begin{array}{l}\text { nasal, auricular, } \\
\text { oral }\end{array}$ & MG-TT-36 \\
\hline Cordia sp. & Boraginaceae & $\begin{array}{l}\text { hulten } \\
\text { taya'e }\end{array}$ & climber & $\begin{array}{l}\text { above } \\
\text { ground }\end{array}$ & harayita & blackleg & cattle & oral, nasal & MG-TT-21 \\
\hline Datura stramonium L. & Solanaceae & gali dimak & herb & fruit, leaf & andelityo & nerve problem & camel & oral & MG-TT-51 \\
\hline \multirow[t]{4}{*}{$\begin{array}{l}\text { Dobera glabra (Forssk.) } \\
\text { Pair. }\end{array}$} & Salvadoraceae & $\begin{array}{l}\text { garsa, } \\
\text { garsaito }\end{array}$ & shrub & leaf & geno & $\begin{array}{l}\text { sudden } \\
\text { sickness }\end{array}$ & $\begin{array}{l}\text { cattle, } \\
\text { goat }\end{array}$ & oral, nasal & MG-TT-20 \\
\hline & & & & leaf & kilem & tick infestation & $\begin{array}{l}\text { cattle, } \\
\text { goat, } \\
\text { camel, } \\
\text { sheep }\end{array}$ & $\begin{array}{l}\text { oral, local on } \\
\text { skin }\end{array}$ & MG-TT-3 \\
\hline & & & & leaf & ladore & blackleg & cattle & nasal, auricular & \\
\hline & & & & leaf & geno & bloat & cattle & oral & \\
\hline \multirow[t]{3}{*}{ Euphorbia sp. } & Euphorbiaceae & engda'eto & shrub & $\begin{array}{l}\text { whole } \\
\text { part }\end{array}$ & sura'ito & CCPP & $\begin{array}{l}\text { goat, } \\
\text { sheep }\end{array}$ & oral, nasal & $\begin{array}{l}\text { MG-TT-6, } \\
\text { MG-TT-52 }\end{array}$ \\
\hline & & & & $\begin{array}{l}\text { whole } \\
\text { part, root }\end{array}$ & $\begin{array}{l}\text { uruga, } \\
\text { kehu }\end{array}$ & diarrhoea & $\begin{array}{l}\text { sheep, } \\
\text { goat }\end{array}$ & oral, nasal & \\
\hline & & & & stem & sengite & CBPP & $\begin{array}{l}\text { cattle, } \\
\text { goat }\end{array}$ & oral, nasal & \\
\hline Grewia villosa Willd. & Tiliaceae & habeleyta & shrub & bark & alhe & $\begin{array}{l}\text { delayed } \\
\text { placenta }\end{array}$ & camel & oral & MG-TT-32 \\
\hline $\begin{array}{l}\text { Heliotropium longiflorum } \\
\text { (ADC. in DC.) Jaub. \& } \\
\text { Spach. }\end{array}$ & Boraginaceae & am'ada & & leaf & indahi & CCPP & sheep & oral & MG-TT-34 \\
\hline \multirow{2}{*}{$\begin{array}{l}\text { Justicia schimperiana } \\
\text { (Hochst. ex Nees) T. } \\
\text { Anders. }\end{array}$} & Acanthaceae & werabikela & shrub & leaf & tele & wound & animals & local on wound & MG-TT-39 \\
\hline & & & & leaf & - & snake bite & animals & oral & \\
\hline $\begin{array}{l}\text { Kanahia laniflora (Forssk.) } \\
\text { R. Br. }\end{array}$ & Asclepiadaceae & $\begin{array}{l}\text { we'a } \\
\text { amhala }\end{array}$ & shrub & leaf & geno & $\begin{array}{l}\text { sudden } \\
\text { sickness }\end{array}$ & $\begin{array}{l}\text { cattle, } \\
\text { goat }\end{array}$ & oral, nasal & MG-TT-27 \\
\hline Kleinia squarrosa Cufod. & Asteraceae & bisilto & shrub & stem & endahi & diarrhoea & goat & oral & MG-TT-42 \\
\hline
\end{tabular}


Table 1 Medicinal plants of the Afar people in Ada'ar District (Continued)

\begin{tabular}{|c|c|c|c|c|c|c|c|c|c|}
\hline Lantana camara L. & Verbenaceae & dat'hara & shrub & leaf & abeb & FMD & cattle & oral & MG-TT-44 \\
\hline Monadenium sp. & Euphorbiaceae & dargudi & shrub & $\begin{array}{l}\text { whole } \\
\text { part }\end{array}$ & haraiti & blackleg & cattle & $\begin{array}{l}\text { oral, nasal, } \\
\text { auricular }\end{array}$ & MG-TT-43 \\
\hline Pergularia tomentosa L. & Asclepiadaceae & ageraboya & shrub & exudate & abeli & babesia & goat & oral & MG-TT-41 \\
\hline \multirow[t]{3}{*}{ Salvadora persica L. } & Salvadoraceae & ada'ito & shrub & leaf & dale & wound & $\begin{array}{l}\text { cattle, } \\
\text { camel, } \\
\text { goat }\end{array}$ & local on skin & MG-TT-18 \\
\hline & & & & leaf & geno & $\begin{array}{l}\text { sudden } \\
\text { sickness }\end{array}$ & $\begin{array}{l}\text { cattle, } \\
\text { goat }\end{array}$ & oral, nasal & $\begin{array}{l}\text { MG-TT-4, } \\
\text { MG-TT-18 }\end{array}$ \\
\hline & & & & $\begin{array}{l}\text { leaf, } \\
\text { above } \\
\text { ground }\end{array}$ & ladore & blackleg & cattle & $\begin{array}{l}\text { oral, nasal, } \\
\text { auricular }\end{array}$ & MG-TT-18 \\
\hline Seddera bagshawei Rendle & Convolvulaceae & bekil tefere & shrub & $\begin{array}{l}\text { whole } \\
\text { part }\end{array}$ & finoita & bloat & $\begin{array}{l}\text { cattle, } \\
\text { goat, } \\
\text { sheep, } \\
\text { camel }\end{array}$ & oral & MG-TT-46 \\
\hline \multirow[t]{3}{*}{ Senna alexandrina Mill. } & Fabaceae & $\begin{array}{l}\text { airogit, } \\
\text { senoyta } \\
\text { meka }\end{array}$ & shrub & leaf & ladore & blackleg & cattle & oral & $\begin{array}{l}\text { MG-TT-13, } \\
\text { MG-TT-35 }\end{array}$ \\
\hline & & & & leaf & sindera & orf & $\begin{array}{l}\text { goat, } \\
\text { sheep }\end{array}$ & oral, nasal & MG-TT-28 \\
\hline & & & & leaf & - & skin infection & donkey & oral & \\
\hline $\begin{array}{l}\text { Sericocomopsis pallida } \\
\text { (S. Moore) Schinz }\end{array}$ & Amaranthaceae & admegarto & shrub & root & gublo & CBPP & cattle & $\begin{array}{l}\text { oral, local on } \\
\text { skin }\end{array}$ & MG-TT-53 \\
\hline $\begin{array}{l}\text { Solanum hastifolium } \\
\text { Hochst. ex Dunal in DC. }\end{array}$ & Solanaceae & askena & shrub & root & abeli & babesia & goat & $\begin{array}{l}\text { oral, nasal, } \\
\text { auricular }\end{array}$ & MG-TT-13 \\
\hline \multirow[t]{2}{*}{ Solanum incanum L. } & Solanaceae & $\begin{array}{l}\text { kolodo'ita, } \\
\text { wakere } \\
\text { ku'us }\end{array}$ & shrub & fruit & kehu & pneumonia & camel & oral & MG-TT-23 \\
\hline & & & & fruit & $\begin{array}{l}\text { goshin, } \\
\text { sura'ito }\end{array}$ & CCPP & goat & nasal & \\
\hline $\begin{array}{l}\text { Trianthema portulacastrum } \\
\mathrm{L} \text {. }\end{array}$ & Aizoaceae & abure & herb & leaf & antibia & $\begin{array}{l}\text { ophthalmic } \\
\text { infection }\end{array}$ & animals & ophthalmic & MG-TT-40 \\
\hline \multirow[t]{2}{*}{$\begin{array}{l}\text { Withania somnifera (L.) } \\
\text { Dunal }\end{array}$} & Solanaceae & $\begin{array}{l}\text { kokerabito, } \\
\text { ubalto }\end{array}$ & shrub & leaf & shelaitu & listeriosis & cattle & oral, nasal & MG-TT-37 \\
\hline & & & & $\begin{array}{l}\text { above } \\
\text { ground }\end{array}$ & ladore & blackleg & cattle & $\begin{array}{l}\text { oral, nasal, } \\
\text { auricular }\end{array}$ & \\
\hline $\begin{array}{l}\text { Ziziphus spina-christi (L.) } \\
\text { Desf. }\end{array}$ & Rhamnaceae & kusra & shrub & leaf & alhe & $\begin{array}{l}\text { delayed } \\
\text { placenta }\end{array}$ & camel & oral & MG-TT-31 \\
\hline- & - & ademudu & shrub & $\begin{array}{l}\text { whole } \\
\text { part }\end{array}$ & sindera & orf & $\begin{array}{l}\text { sheep, } \\
\text { goat }\end{array}$ & oral, nasal & \\
\hline- & - & asahada & tree & $\begin{array}{l}\text { stem, } \\
\text { above } \\
\text { ground }\end{array}$ & kehu & pneumonia & camel & $\begin{array}{l}\text { oral, nasal, } \\
\text { auricular }\end{array}$ & \\
\hline- & Asclepiadaceae & ata'ali & herb & $\begin{array}{l}\text { whole } \\
\text { part }\end{array}$ & haraita & blackleg & cattle & nasal & \\
\hline- & Cucurbitaceae & $\begin{array}{l}\text { kuranda } \\
\text { geita }\end{array}$ & climber & root & aray mude & blackleg & cattle & oral, nasal & MG-TT-55 \\
\hline \multirow[t]{2}{*}{-} & - & muyaito & shrub & branch & $\begin{array}{l}\text { Indahi, } \\
\text { sura'ito }\end{array}$ & CCPP & goat & oral, nasal & \\
\hline & & & & branch & ladore & blackleg & cattle & oral, nasal & \\
\hline- & - & sokoli & shrub & $\begin{array}{l}\text { above } \\
\text { ground }\end{array}$ & geno & $\begin{array}{l}\text { sudden } \\
\text { sickness }\end{array}$ & $\begin{array}{l}\text { cattle, } \\
\text { goat }\end{array}$ & oral, nasal & \\
\hline
\end{tabular}


number of informants who mentioned the plant for any major ailment [14]. According to Trotter and Logan [15], plants which are used in some repetitive fashion are more likely to be biologically active.

\section{Results and discussion}

Medicinal plants reported and ailments treated

A total of 49 medicinal plants were reported to have been used by the Afar people of Ada'ar District for the treatment of different livestock ailments, of which 39 have been identified to a species level (Table 1). Four plants have been identified to genus level and two to a family level. Four plants, for which voucher specimens have not been collected, are only known by their local names. However, attempt has been made to determine the habit of the uncollected plants based on descriptions given by informants. Despite the fact that the study district is an arid land with poor plant cover, a good number of medicinal plants ( 49 species) are being used in the study area to treat various livestock ailments. This could demonstrate the high dependence of the Afar people in the District on ethnoveterinary service as it is cheaper and easily accessible as compared to modern veterinary service. According to Ada'ar District Pastoral, Agriculture and Rural Development Office, there are only three veterinary health posts and two private drug shops in the whole District. Similar study conducted in Tigray Region of Ethiopia revealed the usage of 44 and 60 medicinal plants in Ofla and RayaAzebo districts, respectively, for the treatment of different livestock diseases [7]. Ethnoveterinary studies conducted in Dabat District of the Amhara Region [16] and Chiro District of the Oromia Region in Ethiopia [17] uncovered the use of 18 medicinal plants in each district. A recent report [18] indicated the use of 24 medicinal plants by people in Medebay-Zana District of Tigray Region for ethnoveterinary purpose.

Some of the plants documented during the current study are used elsewhere in Ethiopia to treat same/similar livestock diseases [7,9,19,20]. These include Acacia nilotica (used to treat diarrhoea); Acalypha indica (used against anthrax), Aloe trichosantha (used against anthrax, contagious caprine pleuropneumonia and contagious bovine pleuropneumonia), Balanites aegyptiaca (used against anthrax), Calotropis procera (used against blackleg) and Dobera glabra (used against tick infestation).

The reported medicinal plants belonged to 23 families and 36 genera. Asclepiadaceae and Capparidaceae took the better share of the reported plants (five species each), followed by Euphorbiaceae and Solanaceae (four species each). The families Boraginaceae and Fabaceae had three species each and the families Acanthaceae, Amaranthaceae, Balanitaceae, Cucurbitaceae and Salvadoraceae had two species each. The remaining 12 families had one species each. The fact that Asclepiadaceae and Capparidaceae contributed relatively higher number of medicinal plants might be attribited to better abundance of species in the study area belonging to these families. Kers [21] and Goyder [22] reported that species of the two families are conspicous in drier habitats like that of the study area. The majority (67.3\%) of the reported Afar medicinal plants were shrubs and this could be due to higher abundance of shruby species in the area that better adapt to arid conditions as compared to plants of other life forms. Few were herbs (18.4\%), climbers (8.2\%) and trees (6.1\%).

The plant remedies were prescibed against 23 types of livestock ailments. The majority of the ailments were that of cattle (15), camel (nine) and goat (eight). Six diseases of sheep and one disease of equine were also reported. The higest number of medicinal plants were used to treat blackleg (17 species), contagious caprine pleuropneumonia (eight species), sudden sickness (seven species) and pneumonia (six species) (Table 2). According to unpublished data of Ada'ar District Agriculture and Rural Development Office (ADARD), blackleg and CCPP are among the top five econmically important livestock diseases in the District. The majority of the reported plants were used to treat cattle diseases (32 species), followed by those used against goat (21 species) and camel (12 species) ailments (Figure 2). Based on unpublished data collected in 2009 by ADARD, cattle had the highest population among the livestock in the District, projected to reach 166, 964 heads in 2010.

Most of the medicinal plants (86\%) were used only for curative purpose. However, few (Boscia coriacea, Calotropis procera, Dobera glabra, Salvadora persica and Caralluma sp.) were used for both curative and prophylactic purposes, and one plant, locally known as 'muyaito, was only used for prophylactic purpose.

Table 2 List of livestock diseases against which three or more medicinal have been prescribed

\begin{tabular}{lll}
\hline Disease english name & $\begin{array}{l}\text { Disease } \\
\text { local name }\end{array}$ & $\begin{array}{l}\text { Number of medicinal plants } \\
\text { used in treatment }\end{array}$ \\
\hline $\begin{array}{l}\text { Blackleg } \\
\begin{array}{l}\text { ladore } \\
\text { pleuropneumonia }\end{array}\end{array}$ & 17 \\
$\begin{array}{l}\text { Sudden sickness } \\
\text { Pneumonia }\end{array}$ & geno & 7 \\
Babesia & kehu & 6 \\
Contagious caprine & gublo/ & 5 \\
pleuropneumonia & sengite & 5 \\
Diarrhoea & uruga & 5 \\
Anthrax & geno & 4 \\
Bloat & finoita & 4 \\
External woulnd & dale & 4 \\
Tick infestation & kilem & 3 \\
\hline
\end{tabular}




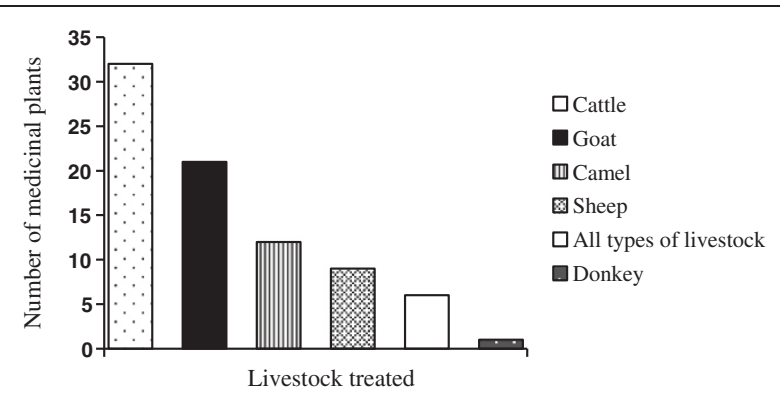

Figure 2 Numbers of medicinal plants used against diffrent livestock ailments.

\section{Parts used and mode of preparation}

Leaf was the most frequently sought plant part accounting for $47 \%$ of the reported medicinal plant species, followed by root $(22 \%)$, whole plant $(16 \%)$ and aboveground part (16\%) (Figure 3). Similarly in studies conducted elsweher in Ethiopia, leaf was indicated to be the most frequently used plant part to treat livestock ailments $[7,18,23]$. A study [24] indicated that collection of leaves poses no significant threat to the survival of individual plants as compared to other parts such as underground part, stem, bark and whole plant.

Most of the remedies (86\%) were processed and used immediately after collection. Few (Acacia nilotica, Aerva javanica, Cadaba glandulosa, Caralluma sp., Grewia villosa and Seddera bagshawei) were reported to be prepared and adminstered while they were fresh or after drying, and one (asahada) was processed and used only after drying. Remedies of 46 medicinal plants (90\%) were

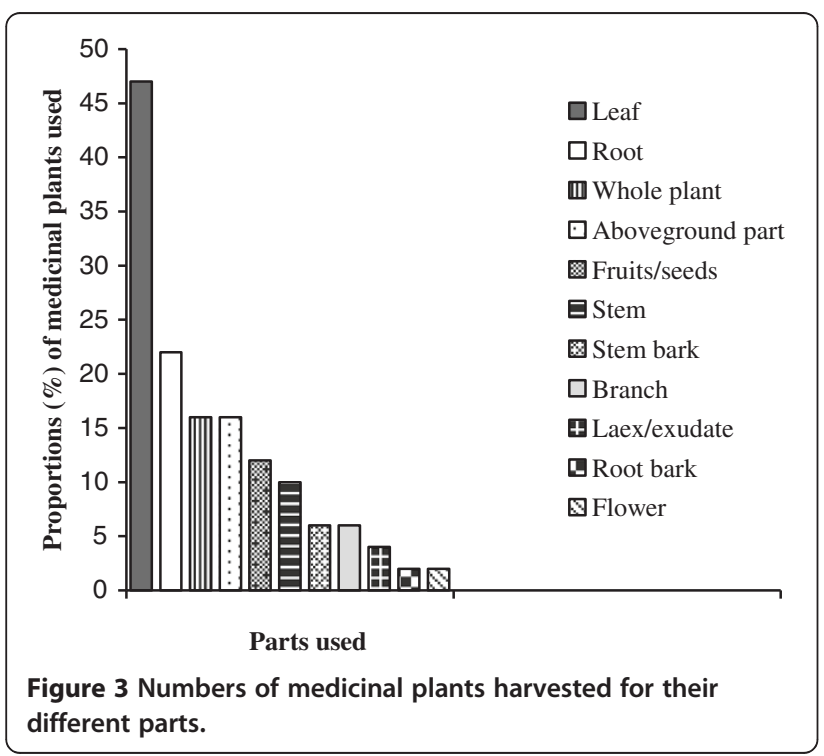

prepared in juice form. Remedies of three species (Acalypha indica, Senna alexandrina and Caralluma sp) were prepared ethier in the form of juice or decocton, and that of two (Kleinia squarrosa and Sericocomopsis pallida) were only prepared in decoction form. There was no pracitice of storing medicinal plants in the study area for future use and this could create shortage of remedies to be used in the District during the dry season when there is scarcity of plants or parts to be harvested.

Preparations of the majority (65\%) of remdies invloved single medicinal plant, which is in agreement with the findings of studies conducted elsewhere in Ethiopia $[23,25]$. However, some (35\%) remedies in the current study area were prepared in a concotion form, by mixing two or more medicinal plants, which is similar to the findings of studies carried out eslewhere in the country $[8,26]$. Many healers in Ethiopia believe that the potency of plant remedies could be enhanced when they are used in concoction form [27].

Water was the most frequently used diluent in the prepartion of remedies accounting for $90 \%$ of the medicinal plants and this could be attributed to either its abundance or to the fact that the active principles contained in most plants are water soluble. Remedies from three species (Boscia coriacea, Calotropis procera and Solanum incanum) were prepared with or without the addition of water, and that of Justicia schimperiana was prepared without the addtion of a diluent. Remedy from one medicinal plant (Pergularia tomentosa) was prepared with the addition of milk as a diluent. Other additives are rarely used. Only butter was reported to be used in the preparation of remedy from Calotropis procera against blackleg.

\section{Route of remedy administration and dosage}

Oral was the most frequently used route of remedy administration accounting for $90 \%$ the medicinal plants, followed by nasal (61\%) and auricular (24\%). Some were adminstred locally on the skin or wound $(12 \%)$ and ophthalmologically (6\%). The dominance of oral application of remedies could be related to the fact that most of the reported health problems are affecting internal organs.

Almost all treatments were given on daily basis, the majority of which $(83 \%)$ were prescribed once a day. Some treatments were given twice $(11 \%)$, trice $(2 \%)$ or four times (2\%) a day. Most treatments (78\%) were reported to be completed within seven days. However, some were $(22 \%)$ known to be given until cure. The amount of remedy taken at one time varied from disease to disease and from informant to informant, even for the same type of ailment. The dose could range from a few drops to one or more litres. Inconsistency of doses have also been reported in similar studies conducted 
elsewhere in the country $[7,17,28]$. All the reported medicinal plants, except one (Balanites aegyptiaca), were found not be toxic even taken at higher doses. The root of Balanites aegyptiaca could kill animals if given in excess during treatment due its toxic effect.

\section{Habitat, avialability and marketablity of medicinal plants} All the medicnal plants used in the study District were uncultivated ones growing in semi-disturbed and distrubed habitats as remnant plants and weeds. There was no tradition or practice by local people to cultivate medicinal plants. Plants were harvested and processed only when needs arised. The use of uncultivated plants is a common practice in Ethiopia [29] and this has been creating an addtional pressure on the populations of wild plants besides to enviromental degradation and deforestation.

Most of the plants were commonly available in the study area and could easily be harvested from sites not very far from homesteads. However, the plants Acacia nilotica, Balanites rotundifolia, Boscia coriacea, Bourreria orbicularis, Euphorbia sp. (engda'eto), Kanahia laniflora, Withania somnifera, 'ademudu', 'asahada', 'muyaito' and 'sokoli' were scarcely available in the study area and, therefore, requred longer time to harvest. None of the medicinal plants that were reported from the study area were available for sale in local markets.

\section{Estimation of medicinal plant healing potential and use- diversity}

Fidelity Level (FL), as an estimation of healing potential, was determined for all reported medicinal plants. Accordingly, Cissus quadrangularis and Solanum incanum were the plants having the highest FL values for their use to treat blackleg and respiratory tract problems, each scoring $100 \%$ (Table 3), followed by Caralluma sp (86\%) and Euphorbia sp. (83\%). According to Trotter and Logan [15], plants

\begin{tabular}{|c|c|c|c|c|}
\hline Plant name & Major ailment group & $I_{p}$ & $I_{u}$ & FL value (\%) \\
\hline $\begin{array}{l}\text { Cissus } \\
\text { quadrangularis }\end{array}$ & Locomotor problems (blackleg) & 4 & 4 & 100 \\
\hline $\begin{array}{l}\text { Solanum } \\
\text { incanum }\end{array}$ & $\begin{array}{l}\text { Respiratory tract problems (CCPP, } \\
\text { pneumonia) }\end{array}$ & 3 & 3 & 100 \\
\hline Caralluma sp & Locomotor (blackleg) & 6 & 7 & 86 \\
\hline Euphorbia sp. & $\begin{array}{l}\text { Respiratory tract problems (CBPP, } \\
\text { CCPP, pneumonia) }\end{array}$ & 5 & 6 & 83 \\
\hline \multirow[t]{2}{*}{$\begin{array}{l}\text { Balanites } \\
\text { aegyptiaca }\end{array}$} & $\begin{array}{l}\text { Digestive system problems (bloat, } \\
\text { colic, diarrhoea) }\end{array}$ & 3 & 5 & 60 \\
\hline & $\begin{array}{l}\text { Septicaemic problems (anthrax, } \\
\text { sudden sickness, trypanosomiasis) }\end{array}$ & 3 & 5 & 60 \\
\hline
\end{tabular}

scoring higher informant consensus values are thought to have better potency as compared to plants with less informant consensus values.

Estimation of Relative Importance (RI) showed Balanites aegyptiaca as having the highest value (1.75), followed by Calotropis procera and Dobera glabra (Table 4), which could indicate the relative abundance of these plants in the study area. Balanites aegyptiaca is used to treat bloat, anthrax, sudden sickness, colic, trypanosomiasis, CCPP and diarrhea. Calotropis procera is used against babesia, blackleg and pneumonia, and as prophylaxis against different ailments. Dobera glabra is used to cure sudden sickness, tick infestation, blackleg and bloat.

\section{Conclusion}

In total, 49 medicinal plants were reported to have been used by the Afar people of Ada'ar District in the Afar Region of Ethiopia for the treatment of different livestock ailments. Leaf was the most frequently used plant part in the preparation of remedies. There was no habit of storing medicinal plants in the study area for future use, and as a result people could face shoratge of supply of parts to be used for remedy prepartion in time of need, especially during the dry season. All the medicnal plants used in the study District were uncultivated ones growing in semi-disturbed and distrubed habitats as as remnant plants and weeds. There was no no habit or practice by local people to cultivate medicinal plants. This could pose a threat to those medicinal plants that are scarecely available in the study area. Efforts, should, therefore, be made to save the scarce medicinal plants of the Ada'ar District from futher depletion through in situ and ex situ conservation methods. Special attention should also be given to those medicinal plants that scored the highest fidelity level values as such plants are thought to have better potencies as compared to those having lower fidelity level values.

Table 4 Relative importance (RI) values for Afar medicinal plants used to treat three or more specific livestock ailments

\begin{tabular}{llll}
\hline Plant name & NP & NCS & RI value \\
\hline Balanites aegyptiaca & 1 & 0.75 & 1.75 \\
Calotropis procera & 0.571 & 1 & 1.571429 \\
Dobera glabra & 0.571 & 1 & 1.571429 \\
Salvadora persica & 0.429 & 0.75 & 1.178571 \\
Euphorbia sp. & 0.571 & 0.5 & 1.071429 \\
Aloe trichosantha & 0.429 & 0.5 & 0.928571 \\
Boscia coriacea & 0.429 & 0.5 & 0.928571 \\
Senna alexandrina & 0.429 & 0.5 & 0.928571 \\
Caralluma sp & 0.429 & 0.5 & 0.928571 \\
\hline
\end{tabular}




\section{Competing interests}

The authors declare that they have no competing interests.

\section{Authors' contribution}

The two authors had significant intellectual contribution towards the design of the study, data collection and analysis and write-up of the manuscript. The authors read and approved the final manuscript.

\section{Acknowledgments}

We would like to thank the Offfice of the Vice President for Research and Dean of Graduate studies of the Addis Ababa University (AAU) for financial support to conduct this study, and the Aklilu Lemma Institute of Pathobiology (ALIPB), AAU, for provision of a field vehicle. We also thank administrators at diffrent levels in Ada'ar District and Abdu Mohammed, local resident, for his translation service throughut the study peripod. We are also grateful to the veterinariams Dr. Tadesse Eguale and Dr. Nigatu Kebede (ALIPB staff members), and Dr. Abdu (veterinarian in Afar Region) for their assistance in translating Afar disease names into English based on signes and symptoms described by informants. Last, but not least, we are highly indebited to informants who were willing to particpate in the study and share their medicnal plant knowledge with us.

Received: 9 August 2012 Accepted: 17 January 2013

Published: 23 January 2013

\section{References}

1. CACC: Ethiopian Agricultural Sample Enumeration, 2001/02 Results for Southern Nations, Nationalities and Peoples' Region. Addis Ababa, Ethiopia: Federal Democratic Republic of Ethiopia, Central Agricultural Census Commission (CACC); 2003.

2. EARO: National Animal Health Research Programme Strategy. Addis Ababa, Ethiopia: Ethiopian Agricultural Research Organization (EARO); 1999.

3. Alemu L: Studies on the Traditional Medicinal Plants of Veterinary Importance in and Around Bahir Dar. DVM thesis: Addis Ababa University; 1993.

4. Tolosa A: Ethnoveterinary knowledge in central highlands of Ethiopia, Sheno, North Shoa. In Proceedings of the $11^{\text {th }}$ Conference of the Ethiopian Veterinary Association: June 1997. Edited by the Ethiopian Veterinary Association. Addis Ababa, Ethiopia; 1997:110-125.

5. Wirtu G, Adugna G, Samuel T, Kelbessa E, Gelato A: Aspects of farmers' knowledge, attitudes and practices of animal health in central Ethiopia. In Proceedings of an International Conference on Ethnoveterinary Medicine, Alternative for Livestock Development: 4-6 November 1997; Pune, India. Edited by Mathias E, Rangnekar DV, McCorkle C.; 1999:41-52.

6. Mesfin T: Ethnoveterinary Practices of Camel Herders of Southern Afar Area. 2000. http://ossrea.net/nw/ethiopia/nw-02.htm.

7. Giday M, Ameni G: An ethnobotanical survey on plants of veterinary importance in two woredas of Southern Tigray, Northern Ethiopia. SINET: Ethiopian J Sci 2003, 26:123-136.

8. Yineger $H$, Yewhalaw D: Traditional medicinal plant knowledge and use by local healers in Sekoru District, Jimma Zone, southwestern Ethiopia. J Ethnobiol Ethnomed 2007, 3:24.

9. Seifu T, Asres K, Gebre-Mariam T: Ethnobotanical and ethnopharmaceutical studies of medicinal plants of Chifra District, Afar region, north eastern Ethiopia. Ethiopian Pharm J 2006, 24:41-58.

10. Philpott J, Abera A, Hadgu K: Livelihood/Emergency Assessment in Afar Region. 2005. http://internal-displacement.org/8025708F004CE90B/\% 28httpDocuments \%29/D6273FE66774116B802570B7005903D2/\$file/Afar + LH-Emer.pdf.

11. CSA: Statistical Abstract 2003. Addis Ababa, Ethiopia: Central Statistical Authority (CSA), Federal Democratic Republic of Ethiopia; 2004.

12. EPA: Afar National Regional State Programme of Plan on Adaptation to Climate Change, 2010. Environmental Protection Authority (EPA) of the Federal Democratic Republic of Ethiopia; 2010. http://www.epa.gov.et/Download/ Climate/Regional\%20Climate\%20Change\%20Adaptation\%20Programmes/ Afar\%20National\%20Regional\%20State\%20\%20Climate\%20Change\% 20Adaptation\%20program.pdf.

13. Bennett $\mathrm{BC}$, Prance GT: Introduced plants in the indigenous pharmacopoeia of northern South America. Econ Bot 2000, 54:90-102.

14. Friedman J, Yaniv Z, Dafni A, Palewitch D: A preliminary classification of the healing potential of medicinal plants, based on the rational analysis of an ethnopharmacological field survey among Bedouins in Negev Desert, Israel. J Ethnopharmacol 1986, 16:275-287.

15. Trotter RT, Logan MH: Informants consensus: a new approach for identifying potentially effective medicinal plants. In Plants in Indigenous Medicine and Diet. Edited by Etkin NL, Bedford H. New York: Redgrave Publishing Company; 1986:91-112.

16. Woldegerima B, Abula T, Ragunathan M: Ethnoveterinary medicinal plants in Dabat District, western Ethiopia. Pharmacognosy Mag 2008, 4:93-99.

17. Bekele A, Musa A: Ethnoveterinary practice in Chiro District, western Hararge, Ethiopia. Pharmacologyonline 2009, 1:128-139.

18. Yirga $G$, Teferi M, Brhane $G$, Amare S: Plants used in ethnoveterinary practices in Medebay-Zana District, Northern Ethiopia. J Med Plants Res 2012, 6:433-438.

19. Giday M, Asfaw Z, Elmqvist T, Woldu Z: An ethnobotanical study of medicinal plants used by the Zay people in Ethiopia. J Ethnopharmacol 2003, 85:43-52.

20. Sori T, Bekana M, Adugna G, Kelbessa E: Medicinal plants in the ethnoveterinary practices of Borana pastoralists, Southern Ethiopia. Int J Appl Res Vet Med 2004, 2:220-225.

21. Kers LE: Capparidaceae. In Flora of Ethiopia and Eritrea. Volume 2, Part 1. Edited by Edwards S, Tadesse M, Demissew S. Addis Ababa, Ethiopia: The National Herbarium, Addis Ababa University and the Department of systematic Botany, Uppsala University; 2000:74-120

22. Goyder DJ: Asclepiadaceae. In Flora of Ethiopia and Eritrea. Volume 4, Part 1. Edited by Hedberg I, Edwards S, Nemomissa S. Addis Ababa, Ethiopia: The National Herbarium, Addis Ababa University and the Department of systematic Botany, Uppsala University; 2003:99-193.

23. Hunde D, Asfaw Z, Kelbessa E: Use and management of ethnoveterinary medicinal plants by indigenous people in 'Boosat', Welenchiti area. Ethiopian J Biol Sci 2004, 3:113-132.

24. Poffenberger M, McGean B, Khare A, Campbell J: Community Forest Economy and Use Pattern: Participatory Rural Apraisal Method in South Gujarat, India. Field Method Manual Volume II. New Delhi: Society for promotion of Wastelands Development; 1992.

25. Giday M, Asfaw Z, Woldu Z: Ethnomedicinal study of plants used by Sheko ethnic group of Ethiopia. J Ethnopharmacol 2010, 132:75-85.

26. Abebe D: Traditional medicine in Ethiopia: the attempts being made to promote it for effective and better utilisation. SINET: Ethiopian J Sci 1986, 9:61-69.

27. Abebe D, Ayehu A: Medicinal Plants and Enigmatic Health Practices of Northern Ethiopia. Addis Ababa, Ethiopia: B.S.P.E; 1993

28. Balemie K, Kelbessa E, Asfaw Z: Indigenous medicinal plant utilization, management and threats in Fentalle area, Eastern Shewa. Ethiopia. Ethiopian J Biol Sci 2004, 3:37-58.

29. Asfaw Z: The role of home gardens in the production and conservation of medicinal plants. In Proceedings of Workshop on Biodiversity Conservation and Sustainable Use of Medicinal Plants in Ethiopia: 28 April-May 2001; Addis Ababa, Ethiopia. Edited by Zewdu M, Demissie A. Addis Ababa, Ethiopia: Institute of Biodiversity Conservation and Research; 2001:76-91.

\section{doi:10.1186/1746-4269-9-8}

Cite this article as: Giday and Teklehaymanot: Ethnobotanical study of plants used in management of livestock health problems by Afar people of Ada'ar District, Afar Regional State, Ethiopia. Journal of Ethnobiology and Ethnomedicine 2013 9:8.

\section{Submit your next manuscript to BioMed Central and take full advantage of:}

- Convenient online submission

- Thorough peer review

- No space constraints or color figure charges

- Immediate publication on acceptance

- Inclusion in PubMed, CAS, Scopus and Google Scholar

- Research which is freely available for redistribution 